\title{
Investigation of urban atmospheric visibility using Haar wavelet transform
}

\section{Chin-Hsiang Luo ${ }^{1 *}$, Chung-Shin Yuan ${ }^{2}$, Che-Yen Wen ${ }^{3}$, Jiun-Jian Liaw ${ }^{4}$ Shih-Hsuan Chiu ${ }^{4}$}

${ }^{1}$ Department of Environmental Engineering, Hung-Kuang University, 34, Chung-Chie Road, Taichung, Taiwan, R.O.C.

${ }^{2}$ Institute of Environmental Engineering, National Sun Yat-Sen University

70, Lien-Hai Road, Kaohsiung, Taiwan, R.O.C

${ }^{3}$ Department of Forensic Science, National Central Police University, 56, Shu-Ren Road, Da-Gang, Guei-Shan, Tao-Yuan, Taiwan, R.O.C

${ }^{4}$ Department of Polymer Engineering, National Taiwan University of Science and Technology, 43, Kee-lung Road, Section 4, Taipei, Taiwan, R.O.C

\begin{abstract}
A method of image processing, Haar wavelet transform, was first applied to establish a new index and provide accurate digital data to present urban atmospheric visibility, instead of the conventional observing method. Digital images of urban areas in Kaohsiung, a city at the south of Taiwan, are analyzed according to brightness (gray level). An index value for a scene image is calculated with a processor of Haar wavelet transform. As a result, a good correlation between the wavelet index values and the visual range values estimated by three trained observers is affirmed by the correlation coefficient $\left(\mathrm{R}^{2}\right)$ of 0.81 . Convenient transmission and the exchangeability of digital images of the real-time landscape facilitate the demonstration of the results on the web site.
\end{abstract}

Keywords: visibility, wavelet transform, image processing, air quality.

\section{Introduction}

Urban atmospheric visibility can influence the overall quality for residents in a geographic area, and the experience of tourists visiting that area. Impaired visibility strongly implies the occurrence of ambient pollution events (Dzubay et al., 1982; Tsai and Cheng, 1997; Yuan and Yang, 1997; Sequeira and Lai, 1998). Therefore, air quality was confirmed to relate with atmospheric visibility (Watson,

\footnotetext{
${ }^{*}$ Corresponding author. Tel: +886-4-26318652; Fax: +886-4-26525245.

E-mail address: andyluo@sunrise.hk.edu.tw
} 
2002). Visibility is scientifically defined as the maximum distance at which the outline of the farthest target can be recognized against a horizon background (Horvath, 1981). This distance (unit: $\mathrm{km}$ ) in atmospheric investigations is the meteorological range of environment. The selected targets, ideally, should be black, an alternative color must be adequately dark in contrast with background. Spacing of selected targets, therefore, restricts the accuracy of the traditional measurements for visibility.

Some models and modified formulas (Williams, et al., 1981; Babson, et al., 1982; Malm and Molenar, 1984; Larson, et al., 1988) for measuring visibility have employed photograph processing techniques to estimate some important factors related to atmospheric visibility: size distribution and scattering properties of suspended aerosols; weather interference; a contrast between targets and background; chemical components of pollutants; etc. In practice, a complicated procedure is not adequate for predicting the real-time atmospheric visibility. Recently, (Luo et al., 2002) applied the physiological definition of Weber's ratio to develop a specific brightness and to calculate the brightness difference between a skyscraper target and its background. A good relationship between the specific brightness values and visual measurements obtained by trained observers was established for visual range of 5-10 km. However, the selection and appearance of an ostensibly perfect target is still a problem in these approaches.

Some applications of image enhancement have been wildly applied in recent years in astronomy, meteorology, aviation and satellite technology, biology, medical analysis, national defense technology, industry, security, geographic information system (GIS), etc. Image enhancement with multi-resolution (or multi-scale) characteristics is an important tool for image recognition. Haar wavelet transform, a popular multi-resolution enhancement method can expand an image to two components (Gonzalez and Woods, 2002). One component is the scaling function, e. g. Haar function, which mathematically creates a serious of discrete functional set. The other component is the wavelet function coding two neighboring signals in an image. The characteristic signal merged in the images of interest can be extracted from different frequency bands for overall scales.

This work reports the development and verification by field investigation of Haar wavelet transform applied to atmospheric visibility measurement of Kaohsiung urban area located at the south of Taiwan. A specific index derived by this transform is adopted to automatically monitor visibility. The effectiveness of this approach is evaluated.

\section{Haar wavelet transform for $2 \mathrm{D}$ images}

Visual recognition by human perception requires brightness (or gray level) change emitted from a target. A high-frequency model (Luo, et al., 2005) illustrates that high-frequency components represent brightness disturbance among all targets in an image. Good visibility is able to appear detailed, and high-frequency components in the scene image increase. The wavelet transform provides good mathematical resolution to localize high-frequency information in an image (Grossmann and Morlet, 1984). The continue wavelet transform of a signal, $f(x)$, is defined by 


$$
\left\langle f(x), \psi_{a, b}(x)\right\rangle=|a|^{-0.5} \int_{-\infty}^{+\infty} f(x) \psi\left(\frac{x-b}{a}\right) d x
$$

where $\langle\cdot$,$\rangle means inner integral operation. a, b \in R$ and $a \neq 0$. The coefficients, a and $b$, represent the dilation and shifting parameters, respectively. In general, $\psi$ is named as the elementary wavelet. Multi-resolution is a basic property of a wavelet theory. For two-dimensional signal, e.g. the digital scene images for urban atmospheric visibility measurements, can be considered as a measurable and integral function, $f(x, y) \in L^{2}\left(R^{2}\right) \cdot L^{2}\left(R^{2}\right)$ represents the vector space built by $f(x, y)$. Multi-resolution calculation is based on the existence of the scaling and wavelet functions, $\phi(x, \mathrm{y})$ and $\psi(x, \mathrm{y})$, in the related sub-spaces, $V_{2^{j}}$ ( Gonzalez and Woods, 2002). A separable scaling function is shown as:

$$
\varphi(x, \mathrm{y})=\varphi(x) \varphi(y)
$$

And the separable wavelet functions with directional sensitivity are

$$
\psi^{h}(x, y)=\psi(x) \varphi(y), \psi^{v}(x, y)=\varphi(x) \psi(y) \text { and } \psi^{d}(x, y)=\psi(x) \psi(y)
$$

$\psi^{h}\left(\psi^{v}, \psi^{d}\right)$ presents signal disturbance of gray level (digital brightness) values along the horizontal (vertical, diagonal) direction in the image. Therefore, a two-dimensional image can be resolved as follows:

$$
\begin{aligned}
& A_{2^{j}} f=\left(\left(f(x, y) * \varphi_{2^{j}}(-x) \varphi_{2^{j}}(-y)\right)\left(2^{-j} n, 2^{-j} m\right)\right)_{(n, m) \in Z^{2}} \\
& D_{2^{j}}^{h} f=\left(\left(f(x, y) * \varphi_{2^{j}}(-x) \psi_{2^{j}}(-y)\right)\left(2^{-j} n, 2^{-j} m\right)\right)_{(n, m) \in Z^{2}} \\
& D_{2^{j}}^{v} f=\left(\left(f(x, y) * \psi_{2^{j}}(-x) \varphi_{2^{j}}(-y)\right)\left(2^{-j} n, 2^{-j} m\right)\right)_{(n, m) \in Z^{2}} \\
& D_{2^{j}}^{d} f=\left(\left(f(x, y) * \psi_{2^{j}}(-x) \psi_{2^{j}}(-y)\right)\left(2^{-j} n, 2^{-j} m\right)\right)_{(n, m) \in Z^{2}}
\end{aligned}
$$

Mapping results of Eqs. (4)-(7) operations are illustrated in Figure 1. The mapping textures represent signal disturbance of brightness extracted from horizontal, vertical and diagonal enhancements in an image. Combination of the scaling function and wavelet function produced by 
Haar transform is used to expand the sampled images for visibility measurements. Haar transform can be presented as a separable and symmetrical matrix $(\mathrm{N} \times \mathrm{N})$ (Gonzalez and Woods, 2002):

$$
A=H f(x, y) H^{T}
$$

A basis function derived from Haar transform is the simplest and orthonormal wavelet (Gonzalez and Woods, 2002) shown as:

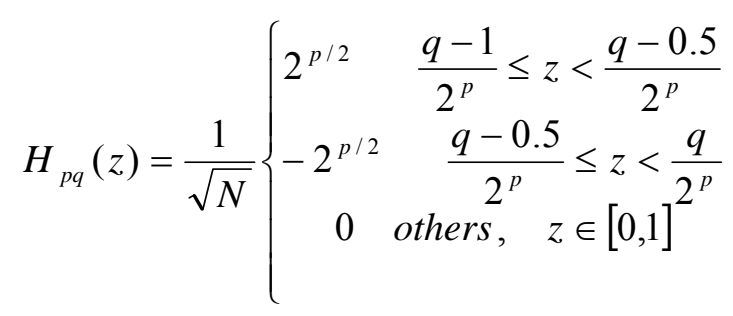

where $N=2^{n}$ and $k=0,1,2, \ldots \ldots \ldots, N-1$. By definition, $k=2^{p}+q-1$. A computing processor is utilized to select a transforming operation and to present the inversed results.

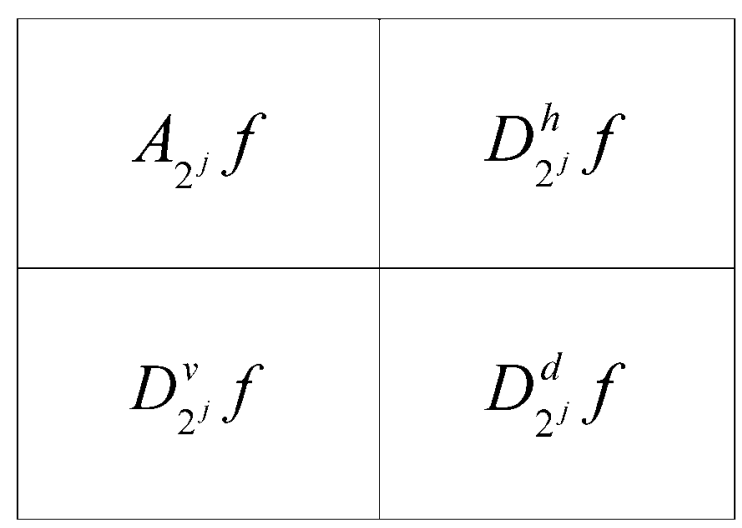

Figure 1. Diagram for the multiresolution mapping of an image.

\section{Application to urban atmospheric images}

\subsection{Image sampling}

The sampling site for this work is on the top of the Linden Hotel which is a famous 42-floor (175 $\mathrm{m}$ height) landmark in the center of Kaohsiung city. Selected targets around this site have been previously orientated by GPS (global positioning system, market type: eTrex LegendT ${ }^{\mathrm{TM}}$ ). These targets include skyscrapers, dark-colored buildings, departments, chimney stacks, the Kaohsiung international airport, schools, gardens, and the boundary line between mountains and sky. The distances between the targets and sampling site range from 0.5 to $15 \mathrm{~km}$. Visual range values (unit: $\mathrm{km}$ ) for visibility measurements were estimated by three trained observers when urban atmospheric 
images were automatically taken by a digital camera (Kodak C 120). To decrease discrepancies among human visual measurements, all observers have participated in the training courses and should pass a test given by an expert at the Kaohsiung weather station. This camera equipped with triple-focus lens and charge-couple device $(C C D)$ can generate a picture quality of $640 \times 480$ pixels. Before monitoring, the response of the $\mathrm{CCD}$ was checked with a standard multicolor chart (a digital image file). In enclosed environment, the camera took a picture of the standard chart lit by an electric bulb with a color temperature of $5000 \mathrm{~K}$. A digital comparison of the photograph of the chart and the chart itself confirmed the constancy of CCD quality. Camera focus was set to infinity ( $\infty)$ to imitate photopic perception of far-gazing human eyes. And camera flash was not applied as images were taken twice between 10 AM and 3 PM daily, from January 1, 2003 to March 1, 2004. Recording and transmission of image acquisition and visual observation results were processed simultaneously via internet and a server installed with Haar wavelet transform operator. Sequentially, wavelet index values for atmospheric visibility were calculated. Instruments adopted and experimental result transmission are schematically shown in Figure 2.

\subsection{Calculation of image brightness}

Brightness difference in an image is the most useful factor for visibility measurements (Middleton, 1963). A digital image for field acquisition provides space dimension and gray level (digital brightness). Gray levels are $2^{\mathrm{k}}$ and estimated by sampling hardware (camera). The resulting image is a k-bit image. All images for urban atmospheric visibility are 8-bit; their gray level values range from 0 to 255 . The gray level value of 0 is set as perfect black. As such, brightness is increasing as the gray level value increases.

Since a color image is produced in three standard components (red, $\lambda=700 \mathrm{~nm}$, green, $\lambda=546 \mathrm{~nm}$, and blue, $\lambda=436 \mathrm{~nm}$ ), any pixel in the color image consists of brightness values for three colors: $\mathrm{L}_{\mathrm{R}}$ (red), $\mathrm{L}_{\mathrm{G}}$ (green) and $\mathrm{L}_{\mathrm{B}}$ (blue). The wavelength of a component is represented by $\lambda$. According to Gonzale and Woods (2002), HSI model describes that humans view a color object by its hue (H), saturation (S), and brightness (intensity, I). Brightness is a subjective descriptor that is practically impossible to measure. Intensity in HSI model is used to present brightness and gray level information. Therefore, a color image is converted into a gray level image (brightness L) with

$$
L=\frac{L_{R}+L_{G}+L_{B}}{3}
$$

Gray level values in an image were collected and mathematically translated to a $M \times N$ matrix containing image elements called pixels (Petrou and Bosdogianni, 1999). 


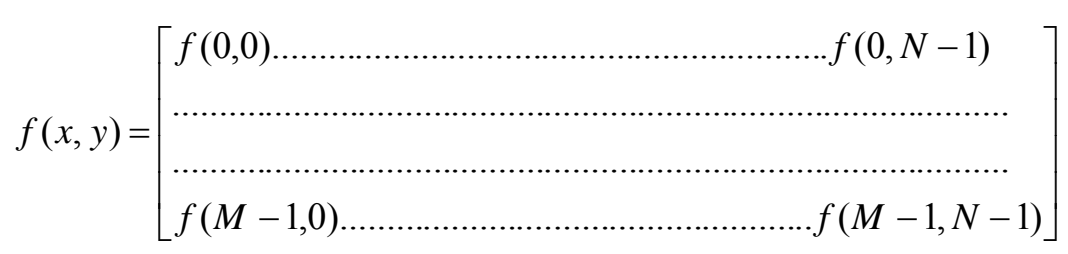

The value in the matrix function is that it presents the gray level at any location of $(\mathrm{x}, \mathrm{y})$.

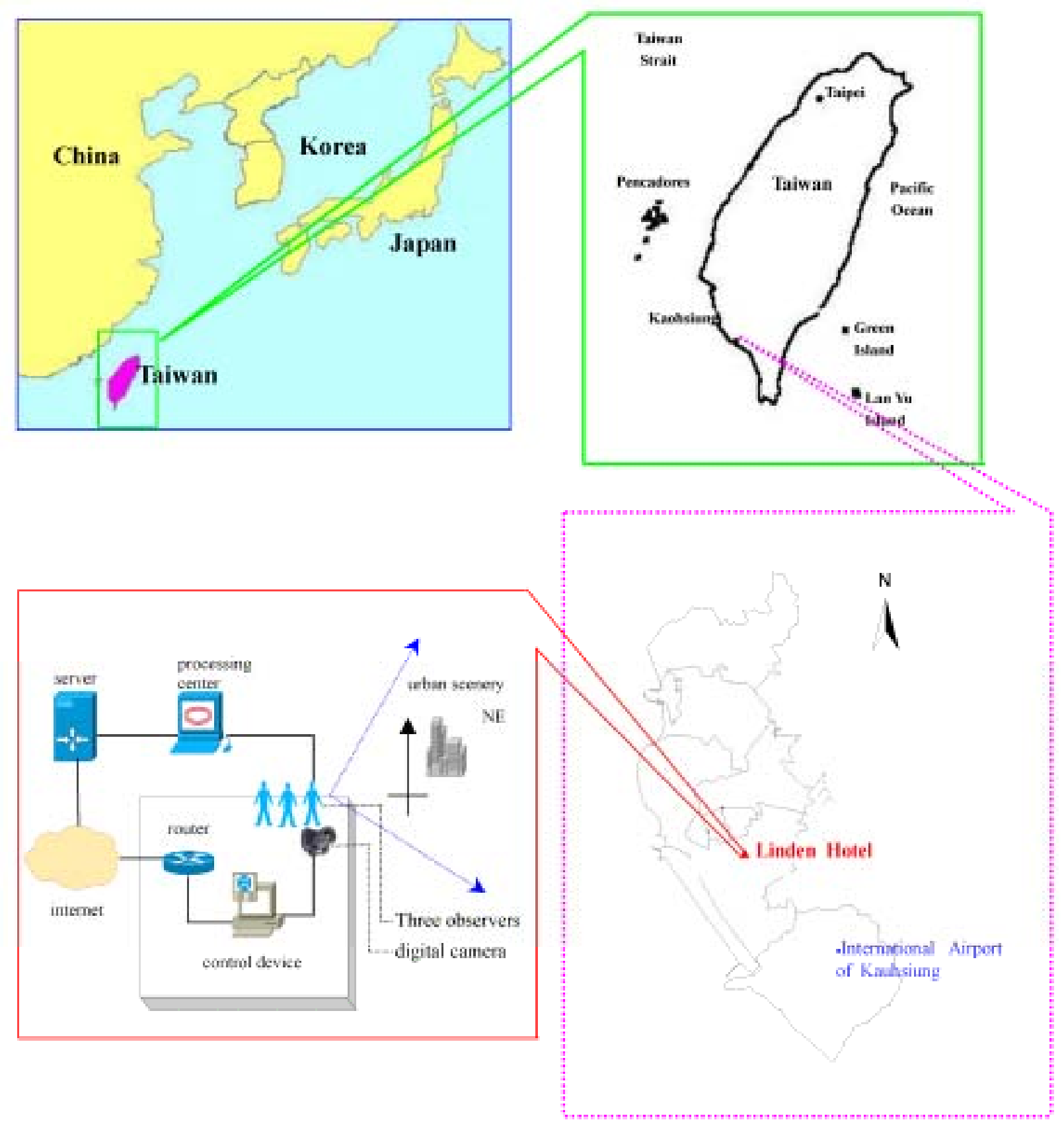

Figure 2. Schema for image processing and sampling location. Icons for computing and data transmission are quoted from Cisco Products. 


\subsection{Development of wavelet index for atmospheric visibility}

Good atmospheric visibility provides a large visual range, as well as presents more signal fluctuation in the scene image. According to the mapping procedure shown in Figure 1, Haar wavelet transform operator estimated the wavelet index value merged in an image taken from the northeast view. For example, it's a clear day with large visual range $(15 \mathrm{~km})$ and good visibility shown in Figure 3(left). No matter horizontal or vertical processing in Eqs. (5) and (6), only one directional frequency gradient merged in an image was confirmed. Diagonal operation expressed in Eq. (7) was able to extract two-directional high-frequency information, therefore, the mean of $D_{2^{j}}^{d} f$ values was selected to be the digital index value for atmospheric visibility. The mapping results extracted from Figure 3(left) were listed in Figure 3(right).

As shown in Figure 4, visual range values estimated by three trained observers are highly correlated with the wavelet index values for the northeast observations. The correlation coefficient $\left(\mathrm{R}^{2}\right)$ reached 0.81 . Our results suggest that human observations for urban atmospheric visibility can be replaced by wavelet index estimation and image acquisition as presented in Figure 2.

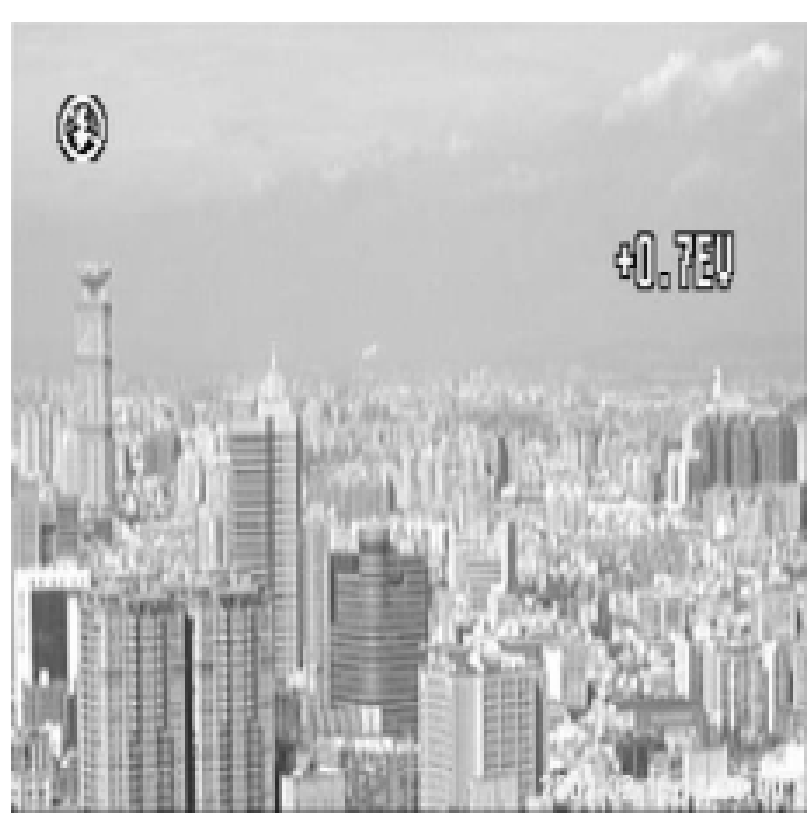

visual range: $15 \mathrm{~km}$

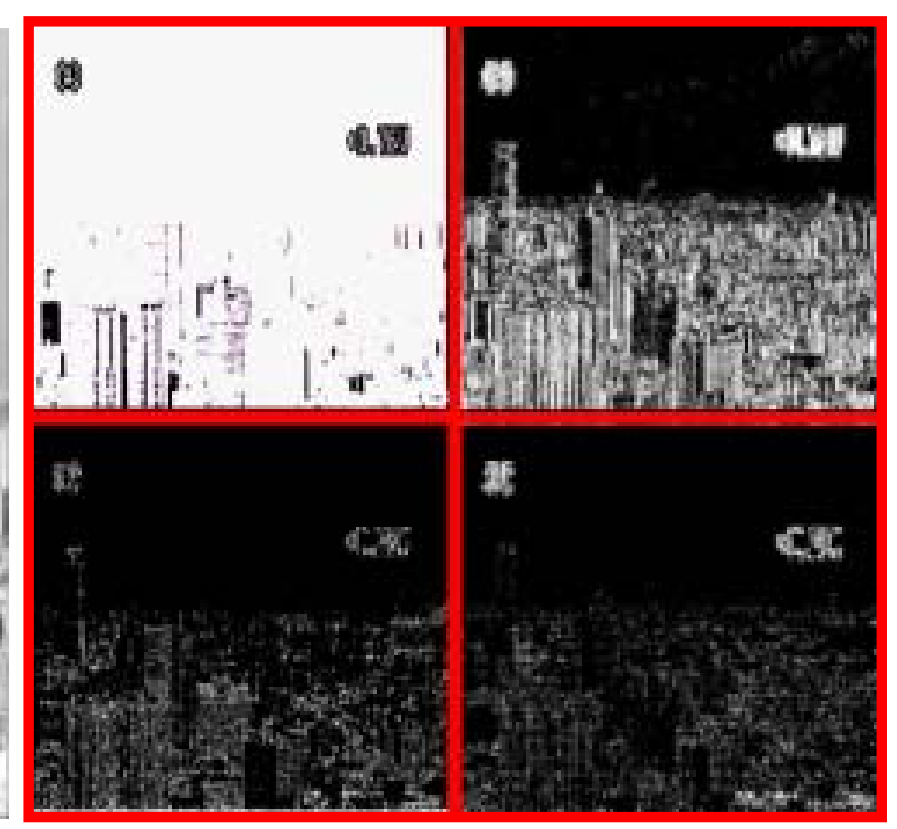

wavelet index: 2.42

Figure 3. An original image (left, visual range $=15 \mathrm{~km}$ ) taken from the northeast view. The mean of wavelet index value derived from the diagonal operation $\left(D_{2^{j}}^{d} f\right)$ of Haar wavelet transform is 2.42 for the mapping image (right). 


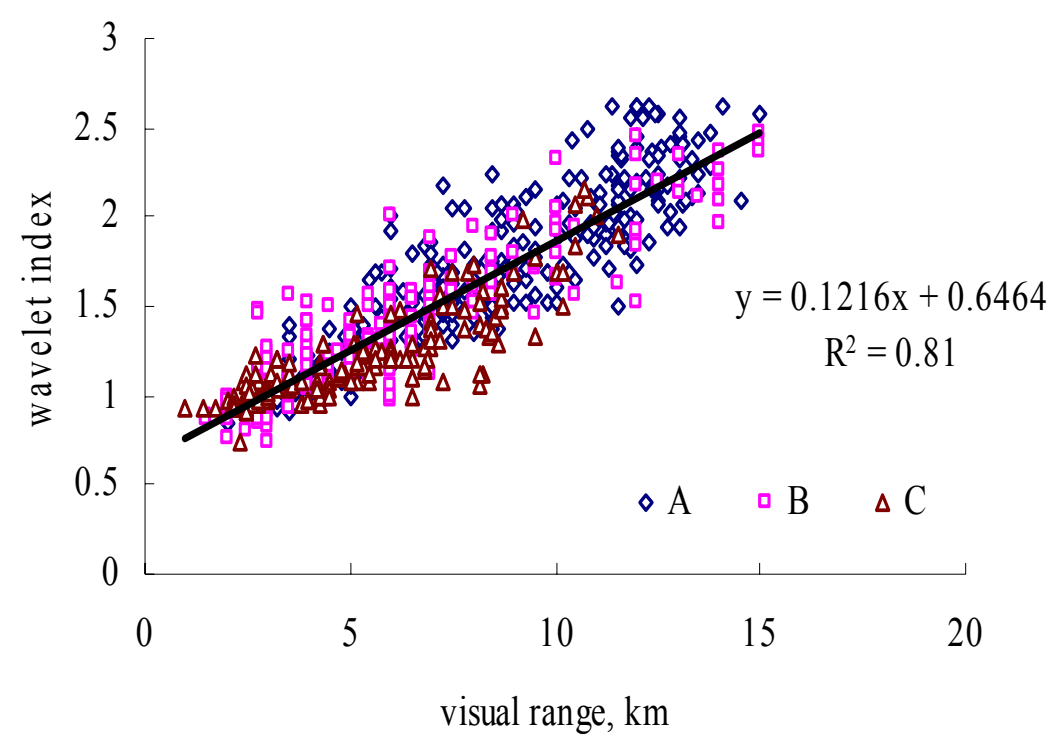

Figure 4. Relationship between the wavelet index values and visual range values detected by three trained observers (A, B, C) for northeast visibility measurements.

\section{Conclusions}

New application of Haar wavelet transform on urban atmospheric visibility was demonstrated to extract high-frequency information from urban atmospheric images, and thus to improve visibility observation methods. Three advancements can be noted. First, an index derived from Haar wavelet transform was used to quantify signal fluctuation merged in a multi-scale image for visibility investigations. Second, a good linear correlation between the index values and visual range values was confirmed for the northeast observations. Third, the real-time visibility recording by weather observers can be replaced by an automatic processor with Haar wavelet transform operator for preventing from human-caused discrepancies. Our practical module of automatically monitoring urban atmospheric visibility is suggested to be further developed for meteorological uses and traffic safety improvement.

\section{Acknowledgment}

The authors would like to thank the National Science Council of Taiwan for financially supporting this research under Contract No. NSC 93 -2211-E-241-002.

\section{References}

Babson, B.L., Bergstrom, R.W., Samuelson, M.A., Seigneur, C., Waggoner, A. and Malm, W.C. (1982), Statistical Analysis of Nephelometer Regional Field Data. Atmos. Environ. 16: 2335-2346. Dzubay, T.E., Steven, R.K., Lewis, C.W., Hern, D.H., Courtney, W.J., Tesch, J.W. and Mason, M.A. 
(1982), Visibility and Aerosol Composition in Houston, Texas. Environ. Sci. Tecnol. 16: 514-525.

Gonzalez, R.C. and Woods, R.E. (2002), Digital image processing, $2^{\text {nd }}$ ed., Pearson Education, Inc, publishing as Prentice Hall.

Grossmann, A. and Morlet, J. (1984), Decomposition of Hardy Functions into Squared Integral Wavelets of Constant Shape. SIAM J. Math. Analysis. 15: 723-736.

Horvath, H. (1981), Atmospheric Visibility. Atmos. Environ.15: 1785-1796.

Larson, S.M., Cass, G.R., Hussey, K.J. and Luce, F. (1988), Verification of Image Processing Based Visibility Models. Environ. Sci. Tecnol. 22: 629-637.

Luo, C.H., Liu, S.H. and Yuan, C.S. (2002), Investigate Atmospheric Visibility by the Digital Telephotography. Aerosol and Air Quality Research. 2: 23-29.

Luo, C.H., Wen, C.Y., Yuan, C.S., Liaw, J.J., Lo, C.C. and Chiu, S.H. (2005), Investigation of Urban Atmospheric Visibility by High-frequency Extraction: Model Development and Field Test. Atmos. Environ, in press.

Malm, W.C. and Molenar, J.V. (1984), Visibility Measurements in National Parks in the Western United States. Journal of the Air Pollution Control Association. 34: 899-904.

Middleton, W.E.K. (1963), Vision through the Atmosphere, University of Toronto Press, Toronto.

Petrou, M. and Bosdogianni, P. (1999), Image Processing: the Fundamentals, John Wiley \& Sons Ltd, England.

Sequeira, R. and Lai, K.H. (1998), The Effect of Meteorological Parameters and Aerosol Constituents on Visibility in Urban Hong Kong. Atmos. Environ.32: 2865-2877.

Tsai, Y.I. and Cheng, M.T. (1997), Relationship between Visibility, Meteorological Factors, and Aerosol Composition in the Taichung Near-shore Area. (1997) International Conference on Aerosol Technology/Environmental Measurement and Control Proceedings, pp. 76-84.

Watson, J.G. (2002), Visibility, Science and Regulation. J. Air\& Waste Manage. Associ. 52: 628-713.

Williams, M., Chan, L. Y. and Lewis, R. (1981), Validation and Sensitivity of a Simulated-photograph Technique for Visibility Modeling. Atmos. Environ. 15: 2151-2170.

Yuan, C.S. and Yang, H.Y. (1997), A Study on the Relationship of Visibility with Suspended Particles and Meteorological Factors in Kaohsiung Metropolitan Area. (1997) International Conference on Aerosol Technology/Environmental Measurement and Control Proceedings, pp. 334-354.

Received for review, December 24, 2004

Accepted, March 10, 2005 